\title{
Improving the Contrast of Breast Cancer Masses in Ultrasound Using an Autoregressive Model Based Filter ${ }^{\star}$
}

\author{
Etienne von Lavante and J. Alison Noble \\ Department of Engineering Science, University of Oxford, UK
}

\begin{abstract}
The assessment and diagnosis of breast cancer with ultrasound is a challenging problem due to the low contrast between cancer masses and benign tissue. Due to this low contrast it has proven to be difficult to achieve reliable segmentation results on breast cancer masses. An autoregressive model has been employed to filter out of the backscattered RF-signal from a tissue harmonic image which is not degraded by harmonic leakage. Measurements on the filtered image have shown a significant (up to $45 \%$ ) increase in contrast between cancer masses and benign tissue.
\end{abstract}

\section{Introduction}

Ultrasonic imaging has become an indispensable tool used in diagnosis of cancer masses during breast cancer diagnosis. However, despite its central role in breast cancer diagnosis, even for skilled radiologists it is still a challenging problem to correctly diagnose and measure cancer masses. This challenge is reflected in the high number of false positive cancer diagnoses made, leading to roughly half of all biopsies made being unnecessary. The main cause of the high uncertainty in ultrasonic cancer diagnosis is strong image artifacts characteristic to ultrasound imagery, such as attenuation, speckle, shadowing and general low contrast.

It is hoped to decrease the uncertainty of the cancer diagnosis with the help of automated medical image segmentation. However, due to the highly variable nature of cancer masses in both shape and image texture, segmentation methods based on statistical priors such as [12] have not shown as much promise as in other areas of medical image analysis. Other recent methods, such as those based on a Bayesian frameworks [34] or local image statistics [5], fail to segment cancer masses reliably. The problem these methods face is that, unlike with cysts, the contrast between cancer masses and surrounding tissue is very low and speckle is the predominant image feature. Therefore one question is whether it is possible to develop an enhancement method specifically aimed at enhancing cancer masses.

Recent advances in signal processing have reduced the effect of speckle on the overall image appearance with the introduction of new techniques such as dynamic beam focussing and tissue harmonic imaging. However, speckle still

\footnotetext{
^ Research supported by UK EPSRC Grant GR-S94575 01.
} 
remains the predominant texture feature in any ultrasound B-mode image. A lot of research has gone into speckle reducing filters, with one of the most recent ones being the Speckle Reducing Anisotropic Filter [6] derived from the Frost and Lee filters [78. Despite their general effectiveness in reducing speckle, they tend to oversmooth the image, and are consequently rarely used for diagnostic breast ultrasound.

For breast cancer diagnosis the only clinically used method which appears to considerably increase the contrast between cancer masses and surrounding tissue is tissue harmonic imaging [9]. This method can be regarded as one of the current state of the art filtering techniques, which is usually implemented by using finite impulse filters (FIR). This therefore suggests that one should take advantage of the principles of tissue harmonic imaging and filtering in the frequency domain to develop a novel filter which will increase the general contrast of breast cancers.

\subsection{Tissue Harmonic Imaging}

Due to the nonlinear nature of tissue, the back scattered signal from an ultrasound pulse interacting with its target will contain, besides the fundamental frequency band of the emitted pulse, also higher harmonics of this band. These higher harmonics are mostly generated due to the peaks of transmitted pulse traveling faster than the troughs due to tissue having different velocities for sound wave propagation in compressed tissue as opposed to relaxed tissue. This effect causes very weak harmonics which are accumulated as the emitted ultrasound pulse propagates through tissue [10]. Hence, the signal received at the ultrasound transducer is made up of the fundamental frequency generated by direct reflections of the ultrasound pulse at tissue interfaces and inhomogeneities, and the higher harmonics generated by the tissue itself, called here the tissue harmonic signal.

There are two principal methods for harmonic imaging, the first one is based on using the pulse inversion technique [1], and the other one is by the application of FIR filters to retrieve the fundamental harmonic and second harmonic from the received signal. While the pulse inversion technique is effective in reducing the effects of harmonic leakage from the fundamental harmonic, it has the major disadvantage of halving the effective frame rate and being more susceptible to motion artifacts. Hence, for tissue harmonic imaging the FIR method is preferred despite its problem of harmonic leakage to the fundamental harmonic affecting the higher harmonics [12, which adds additional noise to these harmonics.

\section{Method}

One way to overcome the effects of harmonic leakage is to develop a linear predictive model of the emitted pulse and then, using parametric spectral estimation, to both estimate the spectral content of the emitted pulse and the remaining tissue harmonic signal. If one has found a good parametric model, the advantages of 
using such a model compared to Fourier Transform based methods is its ability to detect peaks at superresolution, greater resistance to spectral ringing and reduced computational complexity. In several articles [1314 an autoregressive (AR) model of the received RF-signal has been used to estimate various tissue parameters from ultrasound data. The output $y(n)$ of such an AR-model of order $M$ is defined as the output of the following linear filter driven by white Gaussian noise $\eta(n)$ :

$$
y(n)=\eta(n)-\sum_{k=1}^{M} a(k) y(n-k)
$$

where $a(k)$ are the AR coefficients of an order $M$ AR-model. Intuitively, most users of ultrasound devices regard the B-mode image as a two dimensional representation of the power of the reflected signal of an insonified target. With this observation and using the model in (1) one can directly compute the power spectral density (PSD) $P_{y y}(f)$, and consequently the power of the signal, as:

$$
P_{y y}(f)=\frac{r_{p}}{\left\|1+\sum_{k=1}^{M} a(k) e^{-2 j \pi f k}\right\|^{2}}
$$

In linear predictive modeling this model will correspond to an all-poles model, whose power spectrum will be a series of peaks, corresponding to the AR coefficients $a(k)$, among a general flat noise level, whose power is defined by the residual $r_{p}$. The received signal of the emitted pulse can be modelled very closely by an all-poles model, as its power spectrum is, by design, a Gaussian bell-curve. Any noise in the received RF-signal will be due to non-linear interaction of this pulse with tissue, hence corresponding to the signal of the tissue harmonics, as described in the previous section. Using this model, the power of the received tissue harmonic signal is defined by the residue $r_{p}$, which can be directly displayed to yield an estimation of the tissue harmonic signal without the noise introduced by harmonic leakage.

In its current implementation $a(k)$ and $r_{p}$ are estimated from RF-data using Burg's iterative algorithm [15, using an AR-model order of seven, following the recommendation from [13]. Experiments have shown that the results are very robust to the chosen AR-model order. As the resulting residue signal has still a very high dynamic range, the dynamic range has been compressed by applying the Gamma-Brightness correction function, as it is used in analogue screens, to the data before displaying it:

$$
P_{y y \text { new }}=P_{y y} \exp (1 / \gamma) \quad \text { with } \gamma=4.5
$$

The value for the parameter $\gamma=4.5$ was experimentally chosen to give, in the experiment, a sufficient contrast to detect subtle image details in the filtered image. The value for this variable is image dependent and will vary between 3.5 and 5.0 depending on the desired contrast and viewing preferences of the user. 


\section{Methods for Evaluation}

Unfortunately, due to the cross-patient variability of ultrasound data one cannot compare directly the image statistics of ultrasound data across individual image sets. However, despite these variations, the relative difference of statistics measured between cancer regions and "normal" tissue regions remain remarkably similar across multiple data sets. Hence, in order to evaluate any improvements the filtering technique has on the ability to distinguish cancer masses from healthy tissue, the following procedure was used: various image classifiers computed from a region within the cancer mass and a region of healthy tissue with fully developed speckle were compared in each image. For each image, the contrast to noise ratio (CNR) of each classifier was computed as:

$$
C N R=\frac{\left\|\mu_{c}-\mu_{h}\right\|}{\sqrt{\sigma_{c}^{2}+\sigma_{h}^{2}}}
$$

where $\mu_{c}$ and $\mu_{h}$ are the mean of the classifier in the cancer and healthy tissue regions, and $\sigma_{c}$ and $\sigma_{h}$ are the standard deviation of the classifier in the cancer and healthy tissue regions, respectively. Having calculated both the CNR of the filtered image $\mathrm{CNR}_{f}$ and the original B-mode data $\mathrm{CNR}_{b}$, one can calculate the percentage fractional improvement of the classifier frac $_{i m p}$ as:

$$
\text { frac }_{\mathrm{imp}}=\left(\mathrm{CNR}_{f} / \mathrm{CNR}_{b}\right) * 100
$$

\subsection{Intensity Based Statistics}

Using the above method, one can directly compute and compare the general grayscale contrast between cancer masses and healthy tissues. Furthermore, one can also compute the $\mathrm{SNR}$ of both the cancer region $\mathrm{SNR}_{c}$ and healthy region $\mathrm{SNR}_{h}$ by [16]:

$$
\mathrm{SNR}=\bar{I} / \sigma_{I}
$$

with $\bar{I}$ being the mean intensity and $\sigma_{I}$ being the standard deviation of the intensity of the region of interest. However, as the physical principles causing speckle cannot be removed, one should not expect any significant improvement of the SNR by filtering the image, and it will remain by definition close to unity. The main purpose of the SNR is to judge the degree to which speckle is fully developed in the region of interest.

\subsection{Texture Measures}

Due to the texture created by speckle, any trained clinician will not evaluate a region of interest solely on its image intensity, but rather on the general appearance of image texture. Hence, the 1st order statistics give an incomplete picture of the quality of the filtered image. One of the texture based metrics chosen for the validation of this method is the Neighbourhood Gray-Level Difference 
Matrix (NGTDM) [17 and the metrics derived from this statistic. The details on how to populate the NGTDM matrix $s(i)$ can be read in [17. Using this matrix one can calculate the contrast $f_{\text {cont }}$, busyness $f_{\text {busy }}$, complexity $f_{\text {comp }}$ and coarseness $f_{\text {coar }}$ of a texture.

\subsection{Frequency Domain Analysis}

As with all applications of linear predictive modeling, a very important question is the applicability of the employed AR-model to the data. Here the power spectral density (PSD) from a 256 pixel window of a patch with fully developed speckle and of a cancer mass is estimated using a Periodogram. This PSD is then compared to the PSD obtained by estimating (2) over the same data windows.

\section{Results and Discussion}

\subsection{Experimental Set-Up}

Since tissue phantoms cannot reproduce the subtleties of real diagnostic ultrasound data, and the main advantage of harmonic imaging is the ability to identify more subtle features, it was chosen to base all experiments solely on already recorded patient data. All data was recorded during a breast cancer study, on an Analogic AN-2300 with a BK-Medical 8805 probe using a centre frequency of 4.0 $\mathrm{MHz}$, and recording the RF-data at a sampling frequency of $40 \mathrm{MHz}$. From the datasets recorded during this study 25 cancer cases were arbitrarily chosen and analysed using the methods described in Section 3 .

\subsection{Power Spectrum Analysis}

Regarding Fig. 1 proves that the current AR-model indeed estimates with high precision the main harmonics of the backscattered RF-signal. The AR-model also closely follows the frequency shift of the backscattered signal, which is very significant with approximately $2 \mathrm{MHz}$ at the cancer patch, and $1 \mathrm{MHz}$ at the other one. In terms of power, the difference between the AR-model and the Periodogram is larger in the speckle patch than in the cancer mass patch. Hence, using the model from Section 2 one would expect a stronger $r_{p}$ signal from the speckle patch. As this is indeed the case in Fig. 4.3, one can conclude that the model developed here has its validity on real life data, despite the AR-model also following the second harmonic and a non-harmonic peak in the speckle signal.

\subsection{Discussion of Experimental Data}

As can be seen in Fig. 2, when comparing the filtered image to classical Bmode, the quality of the image has been significantly improved. In the filtered image Fig. 4.3 spiculations from the cancer mass are much more pronounced, 


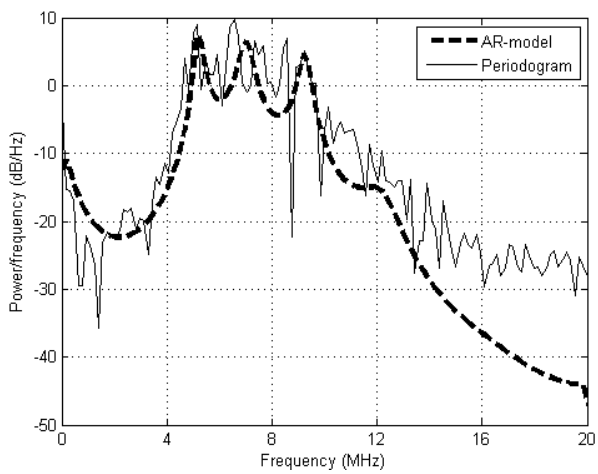

(a)

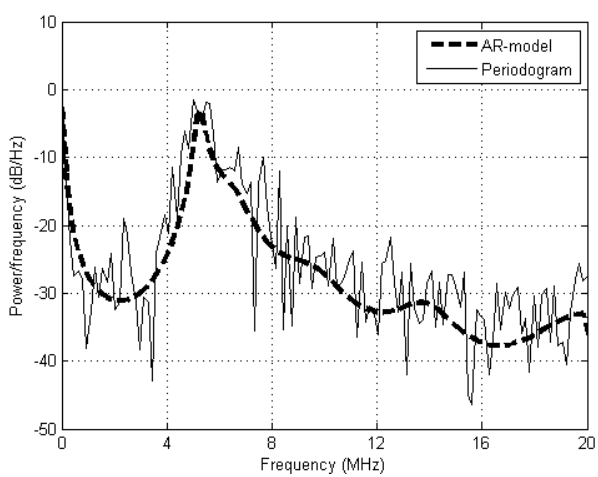

(b)

Fig. 1. comparison of the power spectral density estimated by the employed AR-model and using an Periodogram. Both PSD where computed over 256 data point Hamming window. a) Was computed over a patch with fully developed speckle, b) over a cancer mass.

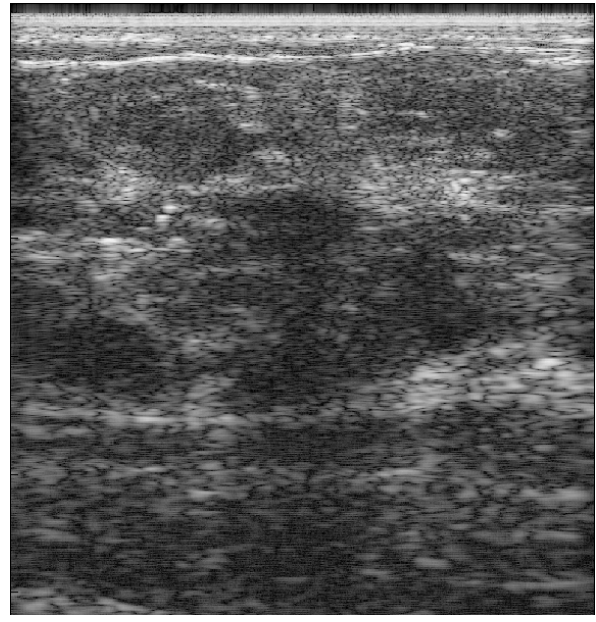

(a)

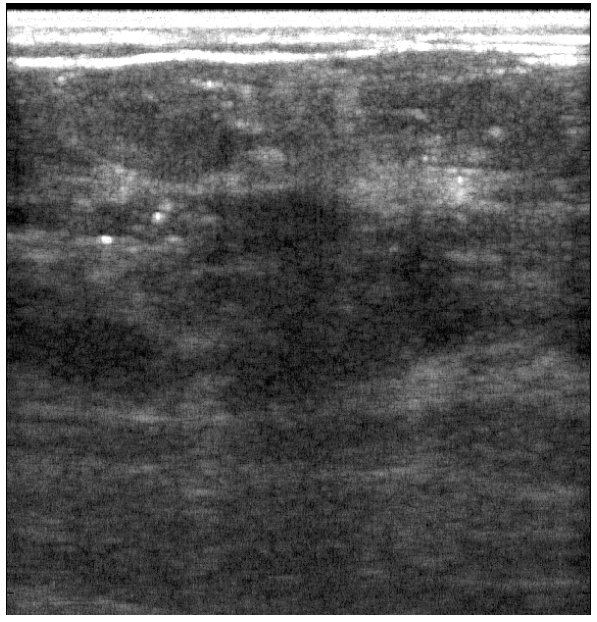

(b)

Fig. 2. the image quality improvement is easily noticable between the classical B-Mode image a) and the filtered image b)

and the overall size of the mass appears a bit larger. As cancer masses tend to appear in ultrasound B-Mode images smaller than their true mass, this difference could be of clinical significance. The visual impression is also strongly supported by the image intensity based statistics, with the fractional improvement of the CNR being on average 145\%. This large improvement in the CNR was found for almost all images. However there were still some images, such as the first set displayed in 2, where there was no improvement. Surprisingly, the SNR improved 
noticeably with an average fractional improvement of the $\mathrm{SNR}_{c}$ being $105 \%$ and the $\mathrm{SNR}_{h}$ being $118 \%$. As it can be seen in Table 1 the results of the texture measures are more ambiguous. Here, only the contrast $f_{\text {cont }}$ and coarseness $f_{\text {coar }}$ classifiers have shown consistent results, as the individual values for the busyness $f_{\text {busy }}$ and complexity $f_{\text {comp }}$ measures have shown a too large variance across the set of images to make any conclusions possible. It appears that these two measures do not well describe the image textures found in ultrasound data. The improvement of the contrast measure of the NGTDM in the filtered was caused by the reduction of speckle inside most cancer regions, producing a very low contrast texture compared to surrounding tissue. The reduction of speckle inside most cancer regions is also responsible for the improvement of coarseness CNR, as the coarseness of the cancer texture in the filtered image has been reduced by a factor of 10 , while the coarseness of the surrounding tissue still remains similar to the values for the B-mode data.

Table 1. Experimental Results

\begin{tabular}{|c|c|c|c|c|c|}
\hline & mean $_{\text {bm }}$ & mean $_{\text {filt }}$ & $\sigma_{\text {bm }}$ & $\sigma_{\text {filt }}$ & frac $_{i m p}$ \\
\hline \hline CNR & 0.817 & 1.187 & 0.052 & 0.129 & $145 \%$ \\
\hline $\mathrm{SNR}_{c}$ & 0.94 & 0.99 & 0.017 & 0.014 & $105 \%$ \\
\hline $\mathrm{SNR}_{h}$ & 0.98 & 1.16 & 0.008 & 0.025 & $118 \%$ \\
\hline
\end{tabular}

\begin{tabular}{|l|c|c|c|}
\hline & CNR $_{\text {bm }}$ & CNR $_{\text {filt }}$ & frac $_{\text {imp }}$ \\
\hline \hline$f_{\text {busy }}$ & 0.226 & 0.49 & $216 \%$ \\
\hline$f_{\text {cont }}$ & 2.65 & 7.89 & $297 \%$ \\
\hline$f_{\text {coar }}$ & 0.094 & 2.387 & $2539 \%$ \\
\hline$f_{\text {comp }}$ & 1.33 & 1.27 & $95 \%$ \\
\hline
\end{tabular}

\section{Conclusion}

Both visual inspection and quantitative analysis have shown that the filtered image has in the majority of the datasets a much higher contrast between the cancer masses and its surrounding tissue. This contrast has shown by visual inspection on many cancer masses an improved ability to discern its shape and size. Regarding the quantitative analysis, the improvement of the image intensity CNR is a notable result. This encourages the possibility of developing a more robust breast cancer segmentation algorithm, which is currently work in progress. The algorithm is not computationally demanding, and could be readily implemented in real time on current hardware. Hence, the next step of our research is to implement this filter on to an ultrasound machine, to assess the degree to which the measured increase in contrast and the subjective image improvement effect the diagnosis of breast cancer in clinical practice. 


\section{References}

1. Xie, J., et al.: Segmentation of kidney from ultrasound images based on texture and shape priors. IEEE Trans. Med. Imag. (1), 45-57 (2005)

2. Zhan, Y., et al.: Deformable segmentation of 3-d ultrasound prostate images using statistical texture matching method. IEEE Trans. Med. Imag. (3), 256-272 (2006)

3. Boukerroui, D., et al.: Segmentation of ultrasound images - multiresoloution 2d and 3d algorithm.... Pattern Recognition Letters (4), 779-790 (2003)

4. Xiao, G.: 3-D Free-hand Ultrasound Imaging and Image Analysis of the Breast. $\mathrm{PhD}$ thesis, University of Oxford (2001)

5. Madabhushi, A., et al.: Combining low-, high-level and empirical domain knowledge for automated segmentation. ... IEEE Trans. Med. Imag. (2), 632-645 (2003)

6. Yu, Y., Acton, S.: Speckle reducing anisotropic diffusion. IEEE Trans. Image Proc. (11), 1260-1270 (2002)

7. Frost, V.S., et al.: A model for radar images and its application for adaptive digital filtering. . . IEEE Trans. Pat. Analy. Mach. Intel. 4(2), 157-165 (1982)

8. Lee, J.S.: Digtial image enhancement and noise filtering by using local statistics. IEEE Trans. Pat. Analy. Mach. Intel. 2(2), 165-168 (1980)

9. Tranquart, F., et al.: Clinical use of ultrasound tissue harmonic imaging. Ultrasound Med. Bio. 25(6), 889-894 (1999)

10. Starritt, H.C., et al.: The development of harmonic distortion in pulsed finiteamplitude ultrasound passing through liver. Physics Med. Bio. 31, 1401-1409 (1986)

11. Simpson, D.H., et al.: Pulse inversion doppler: A new method for detecting nonlinear echoes from mircobubble.... In: Proc IEEE Ultras Symp, pp. 1597-1600. IEEE Computer Society Press, Los Alamitos (1997)

12. Shen, C., Li, P.: Harmonic leakage and image quality degradation in tissue harmonic imaging. IEEE Trans. Ultras. Fer. Freq. Cont. 48(3), 728-736 (2001)

13. Gorce, J., et al.: Processing radio frequency ultrasound images: A robust method for local spectral features estimation... IEEE Trans. Ultra. Fer. Freq. Cont. (12), 1704-1719 (2002)

14. Wear, K.A., et al.: Application of autoregressive spectral analysis to cepstral estimation. .. IEEE Trans. Ultra. Fer. Freq. Cont. (1), 50-58 (1993)

15. Press, W., Teukolsky, S., Vetterling, W., Flannery, B.: Numerical Recipes in C++. Cambridge University Press, Cambridge (2002)

16. Burckhardt, C.B.: Speckle in ultrasound b-mode scans. IEEE Trans Sonics Ultras 25(1) (1978)

17. Amadasun, M., King, R.: Textural features corresponding to textural properties. IEEE Trans Systems Man and Cybern 19(5), 1264-1274 (1989) 